\title{
Roots and Fruits of Decoherence
}

\author{
H. Dieter ZEH \\ Universität Heidelberg \\ www.zeh-hd.de
}

\begin{abstract}
The concept of decoherence is defined, and discussed in a historical context. This is illustrated by some of its essential consequences which may be relevant for the interpretation of quantum theory. Various aspects of the formalism are also reviewed for this purpose.
\end{abstract}

\author{
Contents \\ 1. Definition of concepts \\ 2. Roots in nuclear physics \\ 3. The quantum-to-classical transition \\ 4. Quantum mechanics without observables \\ 5. Rules versus tools \\ 6. Nonlocality \\ 7. Information loss (paradox?) \\ 8. Dynamics of entanglement \\ 9. Irreversibility \\ 10. Concluding remarks
}

\section{Definition of concepts}

The concept of decoherence has become quite popular during the last two decades. However, while its observable consequences have now been clearly confirmed experimentally [1, 2] (see also contributions to this seminar), some misunderstandings regarding its meaning seem to persist in the literature. The phenomenon itself obviously does not depend on any particular interpretation of quantum theory, but its relevance for them may vary considerably 3 , 4. I am indeed surprised about the indifference of most physicists regarding the potential consequences of decoherence in this respect, since this concept arose as a by-product of arguments favoring either a collapse of the wave function as part of its dynamics, or an Everett-type interpretation. In contrast to the Copenhagen interpretation, which insists on fundamental classical concepts, both these interpretations regard the wave function as a complete and universal representation of reality (cf. [5]).

So let me first emphasize that by decoherence I do neither just mean the disappearance of spatial interference fringes in the statistical distribution of measurement results, nor do I claim that decoherence without additional assumptions is able to solve the infamous measurement problem by explaining the stochastic nature of measurements on the basis of a universal Schrödinger equation. Rather, I mean no more (and no less) than the dynamical dislocalization of quantum mechanical superpositions, which are defined in an abstract Hilbert space with a local basis (given by particle positions and/or spatial fields, for example). The ultimate nature of this Hilbert space basis (the stage for a universal wave function) can only be found in a unifying TOE (theory of everything).

Dislocalization arises through the formation of entanglement of any system under consideration (with states $\phi$ ) with another one, such as its unavoidable environment (described by states $\Phi)$. This is often achieved by means of a von-Neumann type "measurement" interaction

$$
\left(\sum c_{i} \phi_{i}\right) \Phi_{0} \rightarrow \sum c_{i} \phi_{i} \Phi_{i} \quad,
$$

which would represent a logical controlled-not operation in the case $i=1,2$ and $\Phi_{0}=\Phi_{1}$. Ideal measurements, that is, those without recoil or change of the state $\phi_{i}$, define "pure decoherence". 
After the interaction, these superpositions still exist, even though they are not there any more [6] 7 . The difference between these two traditionally equivalent phrases reflects the essential character of nonlocal quantum reality. I am indeed convinced that the importance of decoherence was overlooked for the first 60 years of quantum theory precisely because entanglement was regarded just as an aspect of quantum mechanical methods of calculation rather than of physical reality.

Dislocalization of superpositions may be reversible ("virtual") or irreversible in practice ("real" decoherence). In the first case it would either allow the complete relocalization of the superposition ("recoherence"), or at least its reconstruction (the "quantum erasure" of measurement results). The distinction according to the reversibility or irreversibility of decoherence explains also the virtual versus real nature of other "quantum events", such as radioactive decay, particle creation, or excitation. For example, decayed systems remain in a superposition with their undecayed sources until partial waves corresponding to different decay times are decohered from one another. (This has the dynamical consequence of giving rise to an exact exponential decay law see the contribution by Erich Joos to these proceedings.) In contrast to recoherence (complete reversal of the dislocalization), quantum erasure is compatible with the irreversible and non-unitary dynamics of open systems - related to a local entropy decrease at the cost of an entropy increase in the environment [8].

According to (1), dislocalization of superpositions requires a distortion of the environment $\Phi$ by the system $\phi$ rather than a distortion of the system by the environment (such as by classical "noise"). This leads to the important consequence that decoherence in quantum computers cannot be error-corrected for in the usual manner by means of redundant information storage. Adding extra physical quantum bits to achieve redundancy, as it would be appropriate to correct spin or phase flips in the system, would in general even raise the quantum computer's vulnerability against decoherence - for the same reason as the increased size of an object normally strengthens its classicality. (Error correction codes proposed in the literature for this purpose are based on the presumption of decoherence-free auxiliary qubits, which may not be very realistic.)

In special situations, decoherence may be observed as a disappearance of spatial interference fringes. Only for mass points, or center of mass positions of extended objects, are wave functions isomorphic to spatial waves, and only after position measurements with many equivalently prepared objects do they form a statistical distribution. The interference pattern could then conceivable also have been obscured by a slightly varying preparation procedure (for example due to uncontrollable "noise"), while decoherence according to Equ. (1) affects individual quantum states. Because of the latters' nonlocality it leads locally to a reduced density matrix that describes formally an apparent ensemble of states (thus not presuming it). The conceptually important difference between true and apparent ensembles was clearly pointed out by Bernard d'Espagnat 9] when he distinguished between proper and improper mixtures. In the case of virtual (reversible) decoherence, this difference can be observed as recoherence (relocalization of the superposition) - impossible for a proper mixture.

Superpositions thus define pure states, which characterize individual properties that are not present in their formal components. For example, the superposition of two different spinor states is again an individual spinor state (up or down with respect to another direction); the superposition of a $K$-meson and its antiparticle defines a new particle $\left(K_{\text {long }}\right.$ or $\left.K_{\text {short }}\right)$; that of a continuum of positions (in the form of a plane wave) defines a certain "momentum" (wave number). Similarly, a superposition of products of the spin states of two particles (even at different places) by means of Clebsch-Gordon coefficients defines an individual state of total spin, while each particle is then in an "improper mixture" because of its virtual decoherence by the other one. Under unitary transformations (described by a Schrödinger equation) these total states remain pure and can never become ensembles that might represent different measurement outcomes. However, unitary decoherence may irreversibly lead to apparent ensembles (improper mixtures) for local systems, which would precisely explain the required ensembles of measurement outcomes if they were genuine (proper). This consequence can hardly be an unrelated accident! 


\section{Roots in nuclear physics}

Nuclear physics provides some nice examples of many-particle systems which are nonetheless clearly microscopic (found in energy eigenstates). While I was involved in low energy nuclear physics during the sixties, I became irritated by some methods which were quite successfully used. One of them, called the time-dependent Hartree-Fock approximation, describes "stationary" states of heavy nuclei by means of time-dependent determinants of single-nucleon wave functions. But how can the mathematical solution of a static equation $H \psi=E \psi$ know about a concept of time? Similarly, certain deformed nuclei were often described by means of a time-dependent "cranking model" in order to calculate an effective moment of inertia, or to reproduce a Coriolis type coupling between collective rotational states and individual nucleons. However, both parameters characterize the spectra of static energy eigenstates! It turned out that time is here used as a misleading tool to describe static superpositions of one-parametric continua of different determinants in order to construct quantum states for their corresponding collective degrees of freedom (vibrations or rotations around one axis, for example).

For other collective modes, more than one parameter may be required. General rotations, for example, have to be represented by a non-Abelian symmetry group characterized by three Euler angles. Superpositions then assume the form

$$
\Psi=\int d \Omega f(\phi, \theta, \chi) U(\phi, \theta, \chi) \Phi\left(\mathbf{r}_{1}, \ldots, \mathbf{r}_{n}\right)
$$

where $U(\phi, \theta, \chi)$ is the unitary transformation describing a rotation and $d \Omega$ the volume element in this space, while $\Phi$ is a deformed determinant or other "model wave function". There are many other cases where entanglement is classically circumscribed in terms of a time-dependent jargon. Well known is the picture of "vacuum fluctuations" - used to characterize a static state of entangled quantum fields.

If a variational procedure

$$
\delta\langle\Phi|(H-E)| \Phi\rangle=0
$$

with determinants $\Phi$ consisting of single nucleon wave functions $\phi_{i}$, leads to a deformed solution (as it happens for many heavy nuclei), one must first conclude that $\Phi$ can not be an approximation to the correct solution of $H \psi=E \psi$, since it is far from being an angular momentum eigenstate. However, using the degeneracy of these "wrong" solutions under rotations, one may consider their superposition (2) as the next best step. Simultaneous variation of the single-particle wave functions in $\Phi$ and the superposition amplitudes $f(\phi, \theta, \chi)$ then leads to angular momentum eigenstates and rotational spectra, including Coriolis effects for the single particle motion [10].

The superposition (2) may be regarded as being "dislocalized" over all nucleons in such a way that they are all strongly entangled with one another. A strong symmetry violation of the model wave function $\Phi$ may be defined by the quasi-orthogonality of slightly different orientations,

$$
\langle\Phi|U(\phi, \theta, \chi)| \Phi\rangle \approx 0 \quad \text { for } \quad U \neq 1
$$

as though the collective orientation were an observable, and $f(\phi, \theta, \chi)$ therefore the corresponding wave function. In a similar way, phonon degrees of freedom arise in solid bodies. This strong violation of rotational symmetry does not require a "needle limit" of strong geometric asymmetry: it is a collective effect of many slightly asymmetric single-particle wave functions (subsystems). For product wave functions $\Phi=\prod_{i} \phi_{i}\left(\mathbf{r}_{i}\right)$, for example, one would get

$$
\begin{gathered}
\langle\Phi|U(\phi, \theta, \chi)| \Phi\rangle=\prod_{i}\left\langle\phi_{i}|U(\phi, \theta, \chi)| \phi_{i}\right\rangle= \\
\prod_{i}\left(1-\epsilon_{i}\right) \approx \prod_{i} \exp \left(-\epsilon_{i}\right)=\exp \left(-\sum_{i} \epsilon_{i}\right) .
\end{gathered}
$$

(For nucleons, their indistinguishability reduces this result somewhat, and may let nuclei behave as a superfluid.) This quasi-orthogonality is very similar to decoherence, which is often achieved by 
means of a product of inner products of many environmental subsystems (such as many scattered particles) [6]. In lowest approximation of the strong symmetry violation, each nucleon then "feels" only the deformed (apparently oriented) self-consistent potential produced by the others. While there is no absolute orientation in this case of rotational symmetry of the exact Hamiltonian, the latter's dependence on inertial frames allows the nucleons in higher order also to experience a Coriolis-type coupling with the collective angular momentum.

So one may say that the individual nucleons "observe" an apparent asymmetry in spite of the symmetric global superposition of all orientations. However, a similar superposition of different pointer positions occurs in a quantum measurement that is described by von Neumann's unitary interaction (1). This analogy led me to the weird speculation about a nucleus that is big enough to contain a complex subsystem which may resemble a registration device or even a conscious observer. It/she/he would then become entangled with its/her/his "relative world", such as with a definite orientation. Does the dynamical consequence described above then indicate a way to solve the measurement problem? If the nucleons in the deformed nucleus dynamically feel a definite orientation in spite of the global superposition, would an internal observer then not similarly have to become "aware of" a certain measurement result?

This picture was also my first attempt towards a (non-relativistic) quantum cosmology - a kind of Everett interpretation as I later discovered. When I learned about the static WheelerDeWitt quantum universe, described by an equation $H \psi=0$, it also helped me to understand the concept of time that emerges therein (cf. [11] and Sect. 6.2.2 of Ref. [8]). In contrast to a macroscopic body, a nucleus in an energy eigenstate represents a closed quantum "universe". However, it was absolutely impossible at that time to discuss these ideas with colleagues, or even to publish them. An influential Heidelberg Nobel prize winner frankly informed me that any further activities on this subject would end my academic career!

Macroscopic objects are never found in energy eigenstates, but rather in states of certain (usually time-dependent) orientations or positions. Therefore, it was generally concluded that "quantum theory is not made for macroscopic objects" or even the universe. According to Niels Bohr, macroscopic systems have to be described in terms of presumed classical (or "every-day") concepts even though they would have to obey the uncertainty relations.

\section{The quantum-to-classical transition}

Much has been written about the quantum-to-classical transition (cf. [12, 7] and Zurek's contribution to these proceedings). It is evidently crucial for a theory that describes reality exclusively in terms of quantum states, while it would be of no more than secondary importance (such as for explaining the absence of interference patterns in scattering experiments) if classical concepts were presumed for a probabilistic interpretation from the beginning. I could never accept such a fundamental divide between quantum and classical concepts. So one has to understand the different appearance of atoms, nuclei and small molecules on the one hand, and macroscopic objects on the other. If both are described quantum mechanically, their energy spectra differ quantitatively. For example, rotational states of macroscopic objects are very dense. As a consequence, they cannot resist entanglement with their environment even in the case of very weak interactions. Their reduced density matrices must then always represent "mixed states", while the locality of these interactions leads to the vanishing of non-diagonal elements preferentially in the position or "pointer" representation. This is now called decoherence.

Although this term came up more than ten years later (probably it was first used in talks given by Gell-Mann and Hartle at the end of the eighties, preceding their publication of 1993 [13]), I pointed out in a number of papers (see [14, 15]) that this disappearance of certain non-diagonal elements of the density matrix explains superselection rules, which were often postulated as fundamental restrictions of the superposition principle (for example in axiomatic foundations of quantum theory). They were assumed to hold for specific properties, such as electric charge, as well as for "classical observables", although the axioms did not define a precise boundary between quantum and classical concepts.

In these early papers you will not even find the word "entanglement" - simply because this 
concept was so rarely used at that time that I did not know this English translation of Schrödinger's Verschränkung. So I referred to it as "quantum correlations". Remember that even Schrödinger, in his famous paper of 1935 [16], regarded Verschränkung as a mysterious probability relation (which would have to characterize ensembles rather than individual states), since he was convinced that reality has to be defined in space and time.

However, what I had in mind went beyond what is now called decoherence, since it was inspired by the above mentioned picture of an observer inside a closed quantum system. An external observer, who is part of the environment of the observed object, becomes entangled, too, with the property he is observing - just as the observer within the deformed nucleus is entangled with its orientation. He is thus part of a much bigger "nucleus" (or closed system): the quantum universe. So he "feels", or can be aware, only of a definite value of the property he has measured (or separately of different values in different "Everett worlds"). All you have to assume is that his various quantum states which may exist as factor states in these components of the global wave function are the true carriers of awareness. This is even plausible from a quite conventional point of view, since these decohered component states, which are a consequence of the Schrödinger equation, possess all properties required to define observers, such as complexity and dynamical stability (memory). Indeed, these states are the same ones that would arise in appropriate collapse theories if they were, according to von Neumann's motivation, constructed in order to re-establish a psycho-physical parallelism, but I do not see why a modification of the dynamics that eliminates all "other" components from reality should be required.

A genuine collapse that was simply triggered by irreversible decoherence (as recently suggested in a very clever way by Roland Omnès [17]) would not lead to any observable consequences, while other models should either be experimentally confirmed or refuted. As long as they are not confirmed, it is just a matter of taste whether you apply Occam's razor to the facts (by inventing new dynamical laws to cut off what you cannot see) or to the laws (by leaving the Schrödinger equation unchanged) - although this choice must clearly have cosmological consequences (such as a symmetric superposition of many different asymmetric "worlds" that might have been essential, that is, not yet decohered, during early stages of the universe).

For me the most important fruit of decoherence (that is, of a universal entanglement) is the fact that no classical concepts are required any more on a fundamental level. There is then also no need for a fundamental concept of "observables", which would assume certain values only upon measurement - see Chap. 4, and for uncertainty relations then restricting such values. The Fourier theorem for the wave function explains this "uncertainty" in a natural way - well known for classical radio waves, which are themselves real and certain. When Bohr and Heisenberg insisted that the uncertainty relations go beyond the Fourier theorem, they were apparently thinking of spatial wave functions only (thus neglecting entangled states).

For microscopic objects which can be sufficiently isolated, the experimental physicist has a choice between mutually exclusive ("conjugate") measurements, while macroscopic properties are decohered by their unavoidable environment in a general and specific manner. This explains their classical appearance. The corresponding quasi-classical basis in Hilbert space then appears as a classical configuration space, while the conventional "quantization" procedure may be regarded as the re-introduction of these lost superpositions into the (approximately valid) classical theory. Similarly, the classical world appears local to us, since nonlocal entanglement becomes immediately uncontrollable: it is decohered.

In order to illustrate the difference between this and the Copenhagen interpretation, let me quote from a recent publication by Ulfbeck and Aage Bohr from Copenhagen regarding the nature of quantum events. They write [18: "No event takes place in the source itself as a precursor of the click in the counter ...". Hence, there is no decay event in the atom, for example! So far I agree; this conclusion, which is in contrast to earlier interpretations of quantum theory, is enforced by experiments which use reflected decay fragments to demonstrate recoherence (state vector revival) or interference with partial waves resulting from later decay times. In order to appreciate this important change in the Copenhagen interpretation, one may compare the new version with Pauli's claim from the fifties that "the appearance of a certain position or momentum of a particle is a creation outside the laws of nature" (my italics), which clearly refers to the creation of particle 
properties. Ulfbeck and Bohr then continue their sentence of above: "... where the wave function loses its meaning." Here I strongly disagree. After all, it is precisely the arising uncontrollable entanglement with the environment, described by a global wave function, which explains decoherence. These authors are correct, though, when placing the creation of (apparent, I would add) stochastic "events" in the apparatus, where the dislocalization of the relevant superposition becomes irreversible FAPP (for all practical purposes), thus creating an apparent ensemble of quasi-classical wave packets. The dishonesty of the Copenhagen interpretation consists in switching concepts on demand and regarding the (genuine or apparent) collapse as a "normal increase of information" - as though the wave function represented no more than an ensemble of possible physical states. However, this is ruled out by observed state vector revival phenomena.

Of course, you may pragmatically use classical concepts as though they were fundamental even when studying decoherence as a phenomenon. One cannot expect practicing physicists always to argue in terms of a universal wave function. But they may keep in mind that there is a consistent description (thus representing a "quantum reality") underlying their classical terminology. Similarly, a high-energy physicist uses the concepts of momentum and energy to describe the objects in his laboratory, although he knows that they are no more than limited descriptions of the objective relativistic concept of "momenergy". Fortunately, there are other fruits of decoherence in the form of observable phenomena which demonstrate decoherence in action [1, 2. Nonetheless, the derivability in principle of classical (such as particle) concepts undermines the motivation for the Heisenberg picture as well as for Bohm's quantum mechanics, for example.

\section{Quantum mechanics without observables ${ }^{1}$}

In quantum theory, measurements are traditionally described by means of "observables", which in the Heisenberg picture are assumed to replace classical variables, and therefore have to carry the dynamical time dependence. They are formally represented by hermitean operators, and introduced in addition to the concepts of quantum states and their dynamics as a fundamental and independent ingredient of quantum theory. However, even though often forming the starting point of a formal quantization procedure, this ingredient may not be separately required if physical states are universally described by general quantum states (superpositions in an appropriate basis of states) and their dynamics. This interpretation, to be further explained below, would comply with John Bell's quest for a theory in terms of "beables" rather than observables [19. It was for this reason that his preference shifted from Bohm's theory to collapse models (where wave functions are assumed to completely describe reality) during his last years. (Another reason was his antipathy against the "extravagance" - as he called it - of the multiplicity of Everett worlds, which appears in the form of myriads of empty components as well in Bohm's never collapsing wave function.)

Let $|\alpha\rangle$ be an arbitrary quantum state of a local system (perhaps experimentally prepared by means of a "filter" - see below). The phenomenological probability for finding this system in another quantum state $|n\rangle$, say, after an appropriate measurement, is given by means of their inner product, $p_{n}=|\langle n \mid \alpha\rangle|^{2}$, where both state vectors are assumed to be normalized. This transition of state may either correspond to a collapse or a branching of the wave function - though here neglecting the states of the apparatus and the environment. The state $|n\rangle$ represents here a specific measurement. In a position measurement, for example, the number $n$ has to be replaced with the continuous coordinates $x, y, z$, leading to the "improper" Hilbert states $|\mathbf{r}\rangle$. Measurements are called "of the first kind" or "ideal" if the system will again be found in the state $|n\rangle$ (except for a phase factor) whenever the measurement is immediately repeated. Preparations of states can be regarded as measurements which select a certain subset of outcomes for further measurements. $n$-preparations are therefore also called $n$-filters, since all "not- $n$ " results are thereby excluded from the subsequent experiment proper. The above probabilities can be written in the form $p_{n}=\left\langle\alpha\left|P_{n}\right| \alpha\right\rangle$, with a special "observable" $P_{n}:=|n\rangle\langle n|$, which is thus derived from the kinematical concept of quantum states by using their (phenomenological) probabilistic dynamics during measurements, rather than being introduced as a fundamental concept.

\footnotetext{
${ }^{1}$ This chapter is based on Sect. 2.2 of [7. It may be skipped for a quick reading.
} 
Instead of these special " $n$ or not- $n$ measurements" (for fixed $n$ ), one can also perform more general " $n_{1}$ or $n_{2}$ or $\ldots$ measurements", with all $n_{i}$ 's mutually exclusive $\left(\left\langle n_{i} \mid n_{j}\right\rangle=\delta_{i j}\right)$. If the states forming such a set $\{|n\rangle\}$ are pure and exhaustive (that is, complete, $\sum P_{n}=1$ ), they represent a basis of the corresponding Hilbert space. By introducing an arbitrary "measurement scale" $a_{n}$, one may construct general observables

$$
A=\sum|n\rangle a_{n}\langle n|
$$

which permit the definition of "expectation values"

$$
\langle\alpha|A| \alpha\rangle=\sum p_{n} a_{n}
$$

In the special case of a yes-no measurement, one has $a_{n}=\delta_{n n_{0}}$, and expectation values become probabilities. Finding the state $|n\rangle$ during a measurement is then also expressed as "finding the value $a_{n}$ of an observable". A uniquely invertible change of scale, $b_{n}=f\left(a_{n}\right)$, describes the same physical measurement; for position measurements of a particle it would simply represent a coordinate transformation. Even a measurement of the particle's potential energy $V$ is equivalent to an (incomplete) position measurement if the function $V(\mathbf{r})$ is given.

According to this definition, quantum expectation values must not be understood as mean values in an ensemble that represents ignorance about the precise state. Rather, they have to be interpreted as resulting from the probabilities for potentially arising quantum states $|n\rangle$ - regardless of the interpretation of this stochastic process. If the set $\{|n\rangle\}$ of such potential states forms a basis, any state $|\alpha\rangle$ can be represented as a superposition $|\alpha\rangle=\sum c_{n}|n\rangle$. In general, it neither forms an $n_{0}$-state nor any not- $n_{0}$ state. Its dependence on the complex coefficients $c_{n}$ requires that states which differ from one another by a numerical factor must be different "in reality". This is true even though they represent the same "ray" in Hilbert space and cannot, according to the measurement postulate, be distinguished operationally. The states $\left|n_{1}\right\rangle+\left|n_{2}\right\rangle$ and $\left|n_{1}\right\rangle-\left|n_{2}\right\rangle$ could not be physically different from another if $\left|n_{2}\right\rangle$ and $-\left|n_{2}\right\rangle$ were the same state. While operationally indistinguishable in the state $\pm\left|n_{2}\right\rangle$ itself, any numerical factor - such as a phase factor - would become relevant in the case of recoherence. (Only a global factor would thus be "redundant".) For this reason, projection operators $|n\rangle\langle n|$ are insufficient to characterize quantum states.

The expansion coefficients $c_{n}$ relating physically meaningful states - for example those describing different spin directions or different versions of the K-meson - have in principle to be determined (relative to one another) by appropriate experiments. However, they can often be derived from a previously known (or conjectured) classical Hamiltonian theory by means of "quantization rules". In this case, the classical configurations $q$ (such as particle positions or field variables) are postulated to parametrize a basis in Hilbert space, $\{|q\rangle\}$, while the canonical momenta $p$ parametrize another one, $\{|p\rangle\}$. Their corresponding observables,

$$
Q=\int d q|q\rangle q\langle q| \text { and } \mathrm{P}=\int \mathrm{dp}|\mathrm{p}\rangle \mathrm{p}\langle\mathrm{p}|
$$

are then required to obey commutation relations in analogy to the classical Poisson brackets. In this way, they form an important tool for constructing and interpreting the specific Hilbert space of quantum states. These commutators essentially determine the unitary transformation $\langle p \mid q\rangle$ (e.g. as a Fourier transform $\mathrm{e}^{\mathrm{ipq}}$ ) - thus more than what could be defined by means of the projection operators $|q\rangle\langle q|$ and $|p\rangle\langle p|$. This algebraic procedure is mathematically very elegant and appealing, since the Poisson brackets and commutators may represent generalized symmetry transformations. However, the concept of observables (which form the algebra) can be derived from the more fundamental one of state vectors and their inner products, as described above.

Physical states are assumed to vary in time in accordance with a dynamical law - in quantum mechanics of the form $\mathrm{i} \partial_{\mathrm{t}}|\alpha\rangle=\mathrm{H}|\alpha\rangle$. In contrast, a measurement device is usually defined regardless of time. This must then also hold for the observable representing it, or for its eigenbasis $\{|n\rangle\}$. The probabilities $p_{n}(t)=|\langle n \mid \alpha(t)\rangle|^{2}$ will therefore vary with time according to the timedependence of the physical states $|\alpha\rangle$. It is well known that this (Schrödinger) time dependence is formally equivalent to the (inverse) time dependence of observables (or the reference states $|n\rangle$ ). 
Since observables "correspond" to classical variables, this time dependence appeared suggestive in the Heisenberg-Born-Jordan algebraic approach to quantum theory. However, the absence of dynamical states $|\alpha(t)\rangle$ from this Heisenberg picture, a consequence of insisting on classical kinematical concepts, leads to paradoxes and conceptual inconsistencies (complementarity, dualism, quantum logic, quantum information, negative probabilities, and all that). The transition to a Heisenberg picture essentially breaks down for open systems, which cannot obey a Schrödinger equation.

An environment-induced superselection rule means that certain superpositions are highly unstable against decoherence. It is then impossible in practice to construct measurement devices for them. This empirical situation has led some physicists to deny the existence of these superpositions and their corresponding observables - either by postulate or by formal manipulations of dubious interpretation, often including infinities or non-separable Hilbert spaces.

While any basis $\{|n\rangle\}$ in Hilbert space defines formal probabilities, $p_{n}=$ $|\langle n \mid \alpha\rangle|^{2}$, only a basis consisting of states that are not immediately destroyed by decoherence defines "realizable observables". Since the latter usually form a genuine subset of all formal observables (diagonalizable operators), they must contain a nontrivial "center" in algebraic terms. It consists of those which commute with all the rest. Observables forming the center may be regarded as "classical", since they can be measured simultaneously with all realizable ones. In the algebraic approach to quantum theory, this center appears as part of its axiomatic structure 20. However, since the condition of decoherence has to be considered quantitatively (and may even vary to some extent with the specific nature of the environment), this algebraic classification remains an approximate and dynamically emerging scheme.

These "classical" observables thus characterize the subspaces into which superpositions decohere. Hence, even if the superposition of a right-handed and a left-handed chiral molecule, say, could be prepared by means of an appropriate (very fast) measurement of the first kind, it would be destroyed before the measurement may be repeated for a test. In contrast, the chiral states of all individual molecules in a bag of sugar are "robust" in a normal environment, and thus retain this property individually over time intervals which by far exceed thermal relaxation times. This stability may even be increased by the quantum Zeno effect (see [21] for a consistent and exhaustive discussion). Therefore, chirality does not only appear classical in these cases, but also as an approximate constant of the motion that has to be taken into account for defining canonical ensembles in thermodynamics.

The above-used description of measurements of the first kind by means of probabilities for transitions $|\alpha\rangle \rightarrow|n\rangle$ (or, for that matter, by corresponding observables) is phenomenological. However, measurements should be described dynamically as interactions between the measured system and the measurement device. The observable (that is, the measurement basis) should thus be derived from the corresponding interaction Hamiltonian and the initial state of the device. As shown by von Neumann, this interaction must be diagonal with respect to the measurement basis (see (1) and [22]). Its diagonal subsystem matrix elements are operators acting on the quantum state of the device in such a way that the "pointer" evolves into a position appropriate for being read, $|n\rangle\left|\Phi_{0}\right\rangle \rightarrow|n\rangle\left|\Phi_{n}\right\rangle$. Here, the first ket refers to the system, the second one to the device. The states $\left|\Phi_{n}\right\rangle$, representing different pointer positions, must approximately be mutually orthogonal, and "classical" in the explained sense.

Because of the dynamical version of the superposition principle, an initial superposition $\sum c_{n}|n\rangle$ does not lead to definite pointer positions (with their empirically observed frequencies). If decoherence is neglected, one obtains their entangled superposition $\sum c_{n}|n\rangle\left|\Phi_{n}\right\rangle$, that is, a state that is different from all potential measurement outcomes $|n\rangle\left|\Phi_{n}\right\rangle$. This dilemma represents the "quantum measurement problem". Von Neumann's interaction is nonetheless regarded as the first step of a measurement (a "pre-measurement"). Yet, a collapse still seems to be required - now in the measurement device rather than in the microscopic system. Because of the entanglement between system and apparatus, it would then affect the total system. (Some authors seem to have taken the phenomenological collapse in the microscopic system by itself too literally, and therefore disregarded the state of the measurement device in their measurement theory. Such an approach is based on the assumption that quantum states always exist for all systems. This would be in 
conflict with quantum nonlocality, even though it may be in accordance with early interpretations of the quantum formalism.)

If, in a certain measurement, a whole subset of states $|n\rangle$ leads to the same pointer position $\left|\Phi_{n_{0}}\right\rangle$, these states can not be distinguished by this measurement. According to von Neumann's interaction, the pointer state $\left|\Phi_{n_{0}}\right\rangle$ will now be correlated with the projection of the initial state onto the subspace spanned by this subset. A corresponding collapse was therefore formally postulated by Lüders [23] as a generalization of von Neumann's "first intervention" (as he called the collapse dynamics).

In this sense, the interaction with an appropriate measuring device defines an observable. The formal time dependence of observables according to the Heisenberg picture would then describe a time dependence of the states diagonalizing the interaction Hamiltonian, such that, paradoxically, the device would be assumed to be dynamically controlled by the Hamiltonian of the system.

The question whether a certain formal observable (that is, a diagonalizable operator) can be physically realized can only be answered by taking into account the unavoidable environment. A macroscopic measurement device is always assumed to decohere into its macroscopic pointer states. However, as mentioned in Chapter 3, environment-induced decoherence by itself does not solve the measurement problem, since the "pointer states" $\left|\Phi_{n}\right\rangle$ may be defined to include the total environment (the "rest of the world"). Identifying the thus arising global superposition with an ensemble of states (represented by a statistical operator $\rho$ ) that leads to the same expectation values $\langle A\rangle=\operatorname{tr}(\mathrm{A} \rho)$ for a limited set of observables $\{A\}$ would beg the question. This merely operational argument is nonetheless often found in the literature.

\section{Rules versus tools}

As the Everett interpretation describes a "branching quantum world", which mimics a collapsing wave function to the internal observer, the question is often raised for the precise rules of this branching - similar to the dynamical rules for a collapse. Such collapse rules would have to define the individual branches (or the "pointer states") as well as their dynamical probabilities. In contrast, decoherence describes the branching by means of the Schrödinger equation as a dislocalization of initially local superpositions in such a way that the latter become gradually inaccessible to any local observer. Decoherence neither defines nor explains this ultimate (conscious) observer. While the branching is ultimately justified by the observer's locality, the dislocalization itself is an objective dynamical process - in particular occurring in measurement devices.

This unitary dynamical process causes the non-diagonal elements of the reduced density matrices of all dynamically involved local systems (such as those forming a chain of interactions which lead to an observation) to vanish very fast. These indicators of dislocalized superpositions are therefore often used to define decoherence. However, subsystems and their density matrices are no more than convenient conceptual tools, useful because of the locality of all interactions and the causal structure of our world (based on cosmic initial conditions that are responsible for the arrow of time [8]). In contradistinction to a nonlocal superposition, the concept of a density matrix presumes the probability interpretation. The degree of diagonalization of the reduced density matrices would depend on the precise choice and boundaries of subsystems, but this is irrelevant for a sufficient definition of "macroscopically distinct" global branches FAPP. Decoherence may thus be called a collapse without a collapse.

While a genuine collapse theory would have to postulate (as part of the dynamical law) probabilities for its various possible outcomes, in an Everett world all branches are assumed to remain in existence. One can then meaningfully argue only about frequencies of outcomes (such as spots on a screen) in series of measurements that were performed in our branch. Graham was able to show more than thirty years ago [24] that all those very abundant (by number) "maverick Everett worlds" which do not possess frequencies in accordance with the Born probabilities possess a norm that vanishes with increasing size of the series. While their exclusion is nonetheless not a trivial assumption, the norm plays here a similar rôle as phase space does in classical statistical physics: it is dynamically conserved under the Schrödinger equation, and thus an appropriate measure of probability. 


\section{Nonlocality}

Let me continue with another reminiscence from the "dark ages of decoherence" (that ended not before Wojcziech Zurek had published his first papers on this subject in the Physical Review [22]). After I had completed the manuscript for my first paper on what is now called decoherence [14, the only well known physicist who responded to it in a positive way for a long time was Eugene Wigner. He helped me to get it published, and he also arranged for an invitation to a conference on the foundations of quantum theory to be held in Varenna in 1970, organized by Bernard d'Espagnat [25].

When I arrived at Varenna, I found the participants (John Bell included) in hot debates about the first experimental results regarding the Bell inequalities, which had been published a few years before 26. I had never heard of them, but I could not quite share the general excitement, since I was already entirely convinced that entanglement (and hence nonlocality) was a well founded property of quantum states, which in my opinion described reality rather than probability correlations. So I concluded that everybody would now soon agree.

Obviously I was far too optimistic. Some physicists are searching for loopholes in the experiments which confirm the violation of these inequalities until today - even though all experimental results were precisely predicted by quantum theory. Others (perhaps still the majority) are interpreting nonlocality as a "spooky action at a distance", which would have to affect tacitly presumed local quantities or events (such as described by classical concepts). I cannot see anything but prejudice (once shared by Einstein and Schrödinger!) in such an assumption about reality. It is amazing that even Bohm, who did assume the nonlocal wave function to be real, added classical concepts to describe another (local) reality, which would have to include the local observer, and for which the wave function acts as no more than a pilot wave.

It appears strange, too, that certain "measures of entanglement" that have recently been much in use [27] measure only reversible or usable entanglement, while quite incorrectly regarding irreversible entanglement (decoherence) as "noise" or "distortion". It is certainly not an accident that this position appears related to Ulfbeck and Bohr's above-mentioned statement. The observable consequences of Equ. (1) demonstrate that quantum measurements can not be regarded as describing a "mere increase of information" - even in the absence of any recoil. Quantum measurement interactions produce real nonlocal entanglement.

If reality is accepted to be kinematically nonlocal, you also don't need any "spooky teleportation" in order to explain certain experiments that appear particularly attractive to science fiction authors. In all these experiments you have to prepare in advance a nonlocal (entangled) state that contains, in one of its components, precisely what is later claimed to be ported already at its final position. For example, in such a setting two spinors have to be prepared in the form of a Bell state

$$
\begin{gathered}
|\uparrow\rangle_{A}|\downarrow\rangle_{B}-|\downarrow\rangle_{A}|\uparrow\rangle_{B}= \\
|\rightarrow\rangle_{A}|\leftarrow\rangle_{B}-|\leftarrow\rangle_{A}|\rightarrow\rangle_{B}=\ldots \quad,
\end{gathered}
$$

where $A$ and $B$ refer to Alice's and Bob's place, respectively. Nothing has to be ported any more when Alice, say, follows the usual "teleportation" procedure to perform the measurement of another (local) Bell state that includes her spinor of (9) and the one to be ported. Because of the "real" (irreversible) decoherence of the nonlocal superposition (9) caused by this measurement, this initial Bell state becomes an apparent ensemble, such that the entanglement it represents appears to be a statistical correlation from the point of view of all local observers (such as Bob). His apparently incomplete information may then be "completed" by apparently classical means (Alice sending a message to Bob). In quantum terms, this "information transfer" means that Bob and his spin, too, become consistent members of the (partly irreversible) global entanglement (see Joos's Sect. 3.4.2 of [7]). This is then experienced by Bob (in all his arising branches) as a collapse of the wave function. If the relativistic universal Hamiltonian is local (an integral over a spatial Hamiltonian density), it becomes obvious from this unitary treatment that there can be no superluminal influence. Quantum nonlocality is therefore compatible with the dynamical locality of quantum fields that is often referred to as "relativistic causality".

Alice assumes here the rôle of Wigner's well known "friend", who performs a measurement 
without immediately telling the former the outcome (so when is the collapse?). If Pauli's remark of Chap. 5 were right, though, something like telekinesis "outside the laws of nature" would indeed have to create the measurement result at Bob's place in the case of an initial nonlocal Bell state. The term "quantum information" instead of entanglement is therefore quite misleading: entanglement must be part of quantum reality - even though it may often become indistinguishable from a statistical correlation in practice.

Alice would need a similar initial Bell-type superposition of the kind

$$
|C K\rangle_{A}|n o C K\rangle_{B}-|n o C K\rangle_{A}|C K\rangle_{B}
$$

in order to "beam" Captain Kirk (CK) from her to Bob's place, provided he could be shielded against decoherence until she either "measures" his absence at her place or else sends Bob a message to perform a unitary transformation that leads to $|C K\rangle$. (This hypothetical isolation of (10) would indeed permit the existence of a local Schrödinger cat state $|C K\rangle \pm|n o C K\rangle$.) However, the Captain Kirk who is then found at Bob's place could not be one who knows what happened at Alice's place after the preparation of the initial Bell state. You would need a tremendously more complex entangled state, that had to contain all possibilities as part of its nonlocal quantum reality, in order to decide later what to beam. The term "quantum teleportation" drastically misleads the public and should in my opinion not be used by serious scientists. There is enough genuine weirdness that quantum theory has to offer!

In practice, most nonlocal states (except for entanglement between very weakly interacting subsystems, such as photons or neutral spins) would immediately be irreversibly destroyed (that is, become uncontrollable) by decoherence. The apparent locality of our classical world is therefore the consequence of its drastic nonlocality: classical "facts" (or events) appear to be local, although they arise precisely by the dislocalization of superpositions. Hypothetical local (classical) variables which have not been measured are then often regarded as "counterfactuals" (but not rejected as potential concepts even though they would contradict experimental results!), while the nonlocal concepts (superpositions) which successfully describe all these irreversible processes dynamically are simply dismissed as "not conceivably representing reality".

\section{Information loss (paradox?)}

The collapse of the wave function (without observing the outcome) or any other indeterministic process would represent a dynamical information loss, since a pure state is transformed into an ensemble of possible states (described by a proper mixture, for example). The dislocalization of quantum mechanical superpositions, on the other hand, leads to an apparent information loss, since the relevant phase relations merely become irrelevant for all practical purposes of local observers. I will now argue that the "information loss paradox of black holes" (Hawking's lost bet) is precisely based on this decoherence (or otherwise on the collapse of the wave function), and not a specific property of black holes.

For a better understanding one may first consider irreversible processes in classical mechanics, such as Boltzmann's molecular collisions (see Chap. 9). Since they are based on deterministic dynamics (in analogy to quantum unitarity), ensemble entropy is here conserved. However, collisions lead to the formation of uncontrollable statistical correlations, which are irrelevant FAPP in the future. (They are important, though, for the correct backward dynamics because of the specific cosmic initial condition that has to be assumed for this Universe.) This apparent loss (namely, the dislocalization) of information in this classical case affects physical entropy, since this entropy concept disregards by definition the arising uncontrollable correlations [8]. It is defined as an extensive (additive) quantity, usually in terms of "representative ensembles" characterizing the local macroscopic variables, while microscopic (the real) states - including those of subsystems - are objectively determined in principle by the global initial conditions because of the presumed classical mechanical laws. In contrast, quantum mechanical subsystems possess non-vanishing objective entropy (described by improper mixtures) even for a completely defined global state.

In general relativity (GR), "information" may disappear when physical objects fall onto a spacetime singularity, but in classical physics the real state of external matter would still exist and 


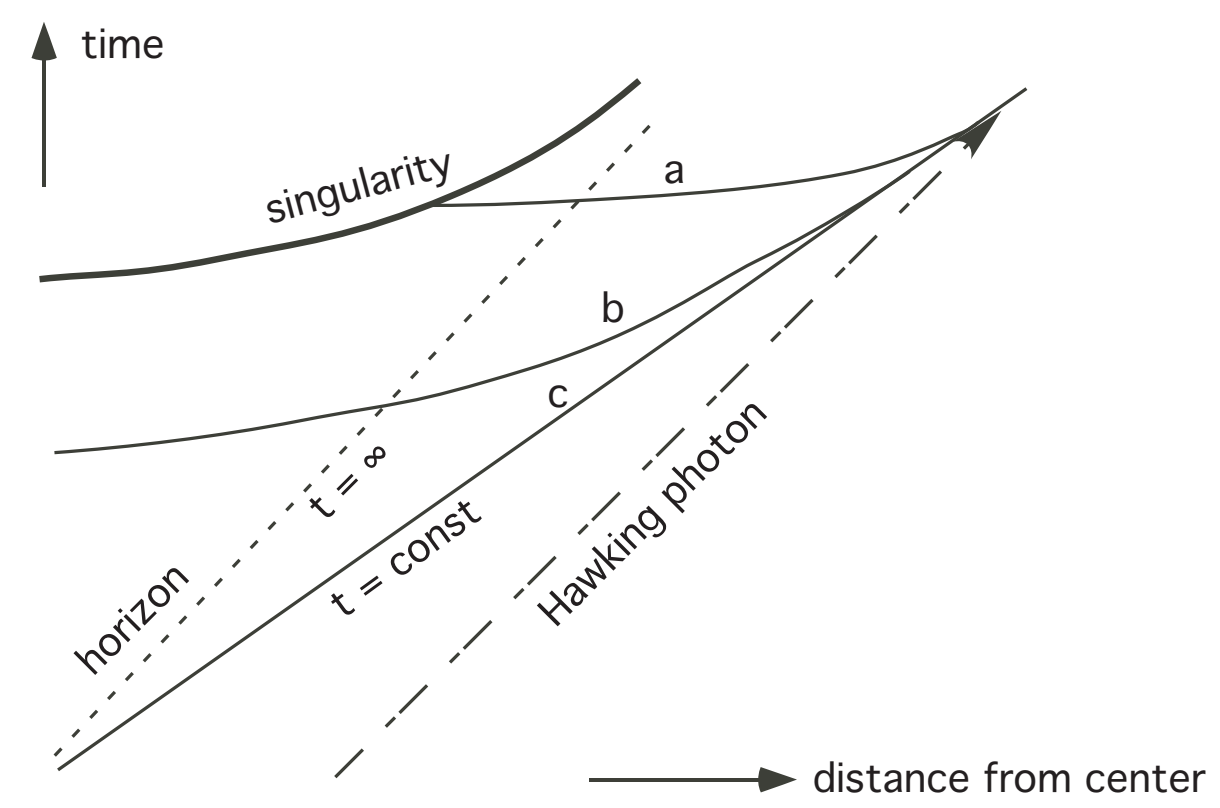

Figure 1: Various simultaneities for a spherical black hole in a Kruskal type diagram: (a) hitting the singularity, (b) entering the regular interior region only, (c) completely remaining outside (Schwarzschild time coordinate $t$ ). Light cones open everywhere at $\pm 45^{0}$ around the vertical time axis in this diagram, while lengths are strongly distorted. Schwarzschild time is appropriate in particular for posing external boundary conditions. The angle between the horizon and the line $t=$ const can here be arbitrarily changed by a passive time translation. This includes the (apparently close) vicinity of the horizon, which can thus be arbitrarily "blown up" in the diagram - thus transforming any Schwarzschild time into the horizontal line $t=0$, for example.

remain determined. In contrast, for quantum mechanics on a classical spacetime, the information loss would have to include all existing entanglement with external matter, thus transforming the latter's improper mixture into a proper one. This conclusion seems to remain true when the black hole disappears by means of Hawking radiation, and this has been regarded as a paradox, since it would violate unitarity.

One may consider the spacetime geometry of a black hole in Kruskal-type coordinates (see Figure 1). Simultaneities used by external observers in asymptotically flat spacetime (such as Minkowski time coordinates in the black hole's rest system) can here be continued towards the center of the spherical black hole in different arbitrary ways. If everywhere chosen according to the Schwarzschild time coordinate $t$, for example, they would never intersect the horizon, but this choice does not affect the density matrix representing the region far from the horizon (far right in the Figure). The information loss noticed by an external observer can therefore not be caused by the singularity - no matter how long he waits. Not even the horizon ever enters his past, and thus never becomes a "fact" for him, while the Hawking radiation which he may observe would originate earlier in Schwarzschild time than the horizon. Because of the singular time dilation, the close vicinity of the horizon can causally affect only the very distant future, since superluminal effects are excluded in spite of quantum nonlocality (cf. Chap. 6).

On the other hand, a macroscopic black hole is permanently affected by various kinds of decoherence [28] - most importantly by means of its retarded radiation. So this quantum radiation must be highly entangled with the remaining black hole, and therefore with all radiation that is emitted later [29]. If usable information about the black hole is stored in the external world (such as in the form of emitted light), it defines separate Everett branches. While the unitary dynamics determines the later global quantum state uniquely, it does not determine an observer's branch: the present state of an observer will have many successors in the future. Any confirmation of the black hole's unitary dynamics would thus require the complete recovery of coherence, including the recombination of Everett worlds - just as it would be required to demonstrate unitarity for 
all macroscopic objects. In practice, their evolution is irreversible. This means that the answer to Hawking's bet has nothing specifically to do with black holes [30].

The spacetime metric with its event horizons and singularities may be assumed to be "real and certain" only in classical GR. In quantum gravity, even the spacetime geometry on which simultaneities are to be defined has to be replaced by an entangled quantum state of matter and geometry [31, 11]. All macroscopic properties are thereby decohered and have to be associated with separate Everett worlds. The Wheeler-DeWitt wave function $\Psi\left[{ }^{3} G, \phi_{\text {matter }}\right]$ (or its generalization to unified theories), which describes their global superposition, defines "probability amplitudes" on all simultaneities (not just on those forming one geometro-dynamic history, that is, a specific foliation of spacetime).

This timeless wave function has to obey certain timeless boundary conditions; it cannot distinguish between past and future singularities 32. For example, these conditions may exclude local singularities (or those with non-vanishing Weyl tensor), or just any entanglement between them and regular regions. This would strongly affect the wave function on all spatial geometries which intersect a black hole horizon, and induce effects corresponding to apparent final conditions. The WKB approximation, which allows quasi-classical spacetime (hence proper times) to emerge by means of the process of decoherence, may then completely break down in such regions of configuration space [33, while the classical spacetime diagram of Figure 1 would lose its meaning close to the horizon.

However, this need not affect quasi-classical solutions restricted to Schwarzschild simultaneities (the external part of the black hole). In particular, Bekenstein's black hole entropy is a general result derived in this region, that cannot be used to confirm specific models of quantum gravity, such as string theory.

\section{Dynamics of entanglement}

The entangled state of any two quantum systems, if assumed to be pure, can always be written as a single sum in the Schmidt canonical form 34]

$$
\psi=\sum_{i} \sqrt{p_{i}} \phi_{i} \Phi_{i},
$$

where the states $\phi_{i}$ and $\Phi_{i}$ forming the two bases are determined (up to linear combinations between degenerate coefficients) by the total state $\psi$. The coefficients can be chosen real and positive by an appropriate choice of phases for the states forming the Schmidt bases, and have therefore been written in the form $\sqrt{p_{i}}$. In contrast to Equ. (1), the states $\Phi_{i}$ are now assumed to be orthogonal: the expansion (11) is thus in general different from the right hand side of (1). This Schmidt representation determines the reduced density matrices in their diagonal form

$$
\begin{gathered}
\rho_{\phi}=\sum_{i}\left|\phi_{i}\right\rangle p_{i}\left\langle\phi_{i}\right|, \\
\rho_{\Phi}=\sum_{i}\left|\Phi_{i}\right\rangle p_{i}\left\langle\Phi_{i}\right| .
\end{gathered}
$$

Since all systems must be assumed to be entangled with their environments, the second system has in principle always to be understood as the "rest of the universe" in order to represent a realistic situation.

If the total state $\psi$ depends on time, the bases $\phi_{i}$ and $\Phi_{i}$ and the coefficients $\sqrt{p_{i}}$ must carry a separate time dependence, which is determined, too, by that of the global state $\psi(t)$. It can be explicitly described [15] by

$$
\begin{array}{r}
\frac{d \sqrt{p_{i}}}{d t}=I m \sum_{j} \sqrt{p_{i}}\langle i i|H| j j\rangle \\
\mathrm{i} \frac{\mathrm{d} \phi_{\mathrm{i}}}{\mathrm{dt}}=\sum_{\mathrm{j} \neq \mathrm{i}}\left(\mathrm{p}_{\mathrm{i}}-\mathrm{p}_{\mathrm{j}}\right)^{-1} \sum_{\mathrm{m}} \sqrt{\mathrm{p}_{\mathrm{m}}}\left[\sqrt{\mathrm{p}_{\mathrm{i}}}\langle\mathrm{ji}|\mathrm{H}| \mathrm{mm}\rangle-\sqrt{\mathrm{p}_{\mathrm{i}}}\langle\mathrm{mm}|\mathrm{H}| \mathrm{ij}\rangle\right] \phi_{\mathrm{i}}
\end{array}
$$




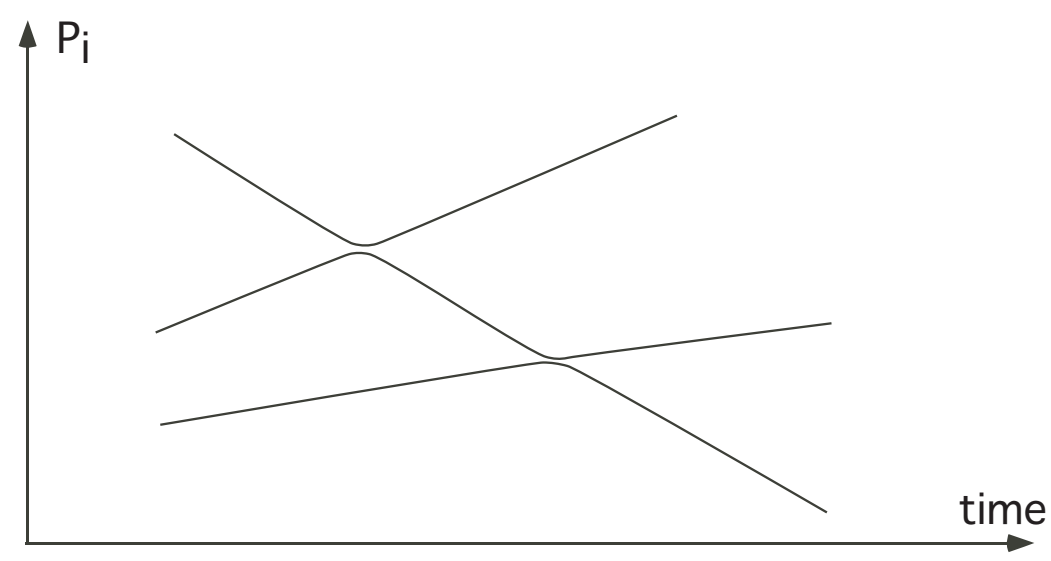

Figure 2: Trajectories of different probabilities $p_{i}(t)$ repel each other, while their corresponding factorizing Schmidt components interchange their identity (including all memories). Causal histories of Schmidt components thus intersect in this diagram, although they never touch. This effect may also be regarded as a pure artifact of the Schmidt representation.

$$
\begin{aligned}
\mathrm{i} \frac{\mathrm{d} \Phi_{\mathrm{i}}}{\mathrm{dt}}=\sum_{\mathrm{j} \neq \mathrm{i}}\left(\mathrm{p}_{\mathrm{i}}-\mathrm{p}_{\mathrm{j}}\right)^{-1} \sum_{\mathrm{m}} \sqrt{\mathrm{p}_{\mathrm{m}}} & {\left[\sqrt{\mathrm{p}_{\mathrm{i}}}\langle\mathrm{ij}|\mathrm{H}| \mathrm{mm}\rangle-\sqrt{\mathrm{p}_{\mathrm{i}}}\langle\mathrm{mm}|\mathrm{H}| \mathrm{ji}\rangle\right] \Phi_{\mathrm{j}} } \\
& +\sqrt{p_{i}} \operatorname{Re} \sum_{m} \sqrt{p_{m}}\langle i i|H| m m\rangle \Phi_{i}
\end{aligned}
$$

The asymmetry between the two subsystems described by $\phi$ and $\Phi$ is here due to an asymmetric phase convention. It could be avoided by a different choice [35].

In classical physics, subsystems would evolve deterministically, controlled by time-dependent Hamiltonians which would depend on the state of the other system (thus forming coupled deterministic dynamics). This classical picture of time-dependent Hamiltonians is often, not very consistently, used also in quantum mechanics - for example in the form of perturbing "kicks" instead of genuine quantum interactions. However, an effective Hamiltonian depending on the state of the environment would require a separately existing state of this environment. In contrast, Equs. (13) define highly nontrivial (not practically usable) nonunitary subsystem dynamics. For this reason, the "probabilities" $p_{i}$ and the entropy $\sum p_{i} \ln p_{i}$ defined by them must usually change in time. In particular, initially separating systems will become entangled.

Although Equs. (13) define a continuous evolution separately for each term of the Schmidt representation, this dynamics seems to be singular whenever two diagonal elements $p_{i}$ of the density matrix become equal. Closer inspection of the dynamics reveals that two eigenvalues coming close repel each other (unless the corresponding matrix elements of the Hamiltonian vanish exactly), and therefore never intersect as functions of time (see Figure 2). Thereby, the quasi-singular dynamics (13) of the states forces the latter to interchange their identity within a very short time. In other words: degenerate probabilities (such as in exact Bell states!) can never occur, while the formal continuity of Schmidt components in Figure 2 is entirely unphysical (not representing any preserved identity of states). Subsystem density matrices are not affected by this phenomenon, since the resonance terms are a consequence of the ambiguity of their degenerate eigenstates. The nonunitary dynamics of entangled density matrices can implicitly (that is, depending on the solutions of (13)) be written as 36

$$
\begin{aligned}
i \frac{d \rho_{\Phi}}{d t} & :=i \frac{d \sum p_{i} \Phi_{i} \Phi_{i}^{*}}{d t} \\
& =\sum_{i, j}\left(\sqrt{p_{i}}\langle i j|H| \psi\rangle-\sqrt{p_{j}}\langle\psi|H| j i\rangle\right) \Phi_{i} \Phi_{j}^{*}
\end{aligned}
$$

Initially separating (factorizing) states are of special interest for the process of decoherence. While this assumption enforces an initial degeneracy to exist between all vanishing probabilities, 
the initial component with $p_{0}=1$ would at least quadratically depend on time because of the time reversal symmetry of the global Schrödinger equation. In this small-times approximation its precise form can be derived by means of perturbation theory as

$$
p_{0}(t) \approx 1-t^{2} A,
$$

where the quantity

$$
A=\sum_{j \neq 0, m \neq 0}|\langle j m|H| 00\rangle|^{2}
$$

has been called a deseparation parameter. It measures the arising entanglement (that is, the growing deviation from separating states). Index pairs $j m$ here refer to product states $\phi_{j} \Phi_{m}$. Note that $A$ is different from the quantity

$$
B=\sum_{j m \neq 00}|\langle j m|H| 00\rangle|^{2} \geq A,
$$

which measures the total change of the global state in this approximation (including the "classical" change characterizing a time-dependent product). If the environment and the interaction Hamiltonian $H$ are given, the deseparation parameter $A$ can be used to estimate the robustness of certain states against decoherence. For example, coupled harmonic oscillators turn out to be robust when in coherent states (such as in states describing classical fields), while their energy eigenstates (such as photon number eigenstates) are unstable against decoherence 15] (a result that was later confirmed in [37]).

\section{Irreversibility}

The dynamics of entanglement, derived from a global Schrödinger equation and discussed in the preceding chapter, is time reversal invariant. An asymmetry may be introduced by assuming initially separating states, for example by using Equ. (15) exclusively for $t>0$. However, if one of the two systems is indeed the "rest of the universe", this assumption can be exact only once (such as at the big bang), although similar assumptions may then approximately apply later, too, in particular for dynamically autonomous Everett components after they have branched off (or, alternatively, after a time-asymmetric collapse).

A similar situation of special initial conditions is known to be required for irreversible processes in general. For example, Boltzmann collisions are assumed to affect initially uncorrelated single particle distributions (defined in $\mu$-space), although correlations must have built up ever since the big bang. The point here is that these correlations remain irrelevant in the future because of the chaotic nature of these classical systems and the enormous lengths of their Poincaré recurrence times reflecting a very large information capacity of correlations. Decoherence produced by scattering of photons or molecules [ 6 is analogous to Boltzmann's entropy production by means of molecular collisions, while coupled oscillators, which have often been used to study decoherence 38, may be useful because of their analytical solutions, but are known to possess pathological properties: they are not mixing in a thermodynamical sense if treated as closed systems. Similar arguments apply to systems consisting of interacting spins, which may better be regarded as representing virtual decoherence - in strong contrast to systems which would classically show chaotic behavior [39.

In quantum theory, reversibility would not even hold in principle in the case of a genuine stochastic collapse of the wave function. If the Schrödinger equation is instead assumed to be universally exact, recoherence of different Everett branches would have to be taken into account in order to facilitate reversibility. This can be excluded FAPP (just as the reversal of any other macroscopic arrow of time), and it is therefore neglected in the usual description of phenomena by means of irreversible master equations, for example.

This general mechanism of irreversible dynamics was elegantly formulated by Zwanzig 40] by means of idempotent operators $P$ acting either on classical statistical probability distributions or on density matrices $\rho$. These "Zwanzig projectors" need neither be linear nor hermitean. They are chosen to formalize the neglect of an appropriate kind of "irrelevant information". Idempotence 


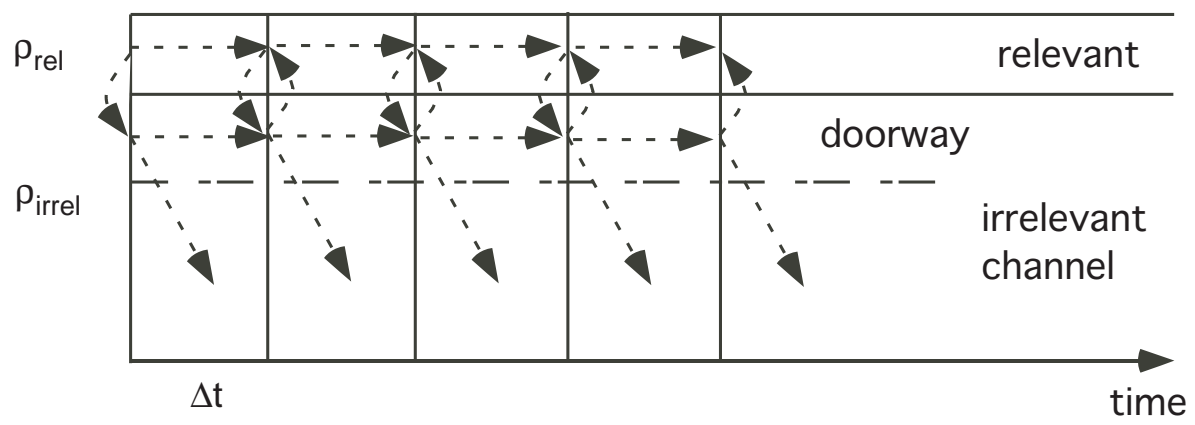

Figure 3: Propagation of "information" contained in the statistical distribution or density matrix $\rho$ for the case $\rho_{\text {irrel }}(t=0)=0$. It propagates through two coupled "channels" (relevant and irrelevant - see also Figs. $3.2-3.4$ of [8]). Intervals $\Delta t$ characterize steps of integration for the coupling $P H(1-P)+(1-P) H P$ in the interaction representation. The entropy, defined in terms of $\rho_{\text {rel }}$, grows irreversibly FAPP if relevant information disappears into the irrelvant channel for very long Poncaré times (usually by far exceeding the age of the universe). This may allow the formulation of master equations for $\rho_{r e l}$. If the irrelvant channel describes entanglement, the irreversible loss represents real decoherence, while the "doorway channel", which is part of the irrelvant channel, corresponds to virtual decoherence.

$\left(P^{2}=P\right)$ means that throwing away the same information twice at the same time does not add anything to doing it once. One should keep in mind, however, that density matrices usually represent improper mixtures, such that a dynamically applied Zwanzig projection includes collapse dynamics, while it would merely represent dynamical mixing for statistical distributions.

A linear Zwanzig projection is given by tracing out the environment,

$$
\rho_{\text {rel }}=P_{\text {sub }} \rho=\rho^{(\phi)}=\operatorname{Trace}_{\Phi} \rho,
$$

while a nonlinear one may merely neglect correlations between the two subsystems,

$$
\rho_{\text {rel }}=P_{\text {sep }} \rho=\rho^{(\phi)} \rho^{(\Phi)}=\operatorname{Trace}_{\Phi} \rho \operatorname{Trace}_{\phi} \rho
$$

There are many other applications of this very general concept, which can also be regarded as a generalized coarse graining. For example, one may just neglect quantum correlations (entanglement), while leaving all classical correlations intact. As another example, Boltzmann's Stoßzahlansatz neglects dynamically all arising statistical correlations between particles.

Zwanzig projectors are useful, in particular, since they often allow the formulation of an effective (approximately autonomous) dynamics for $\rho_{\text {rel }}$. This requires the special initial, but approximately maintained, condition $\rho_{\text {irrel }} \approx 0$, which may lead to a master equation of the form

$$
\frac{d \rho_{r e l}}{d t}=-G_{r e t} \rho_{r e l},
$$

applicable in the forward direction of time. $G_{r e l}$ must be a positive operator, acting on the density matrix, in order to describe an entropy increase (loss of information). The entropy is here defined by

$$
S=-k_{B} \operatorname{Trace}\left[\rho_{\text {rel }} \ln \rho_{\text {rel }}\right] \quad .
$$

This dynamics is schematically depicted in Figure 3 (cf. Chap. 3 of $[\underline{8}$ ), where relevant information is permanently lost to the "irrelvant channel". For the Zwanzig projector $P_{\text {sep }}$ this process describes the production of entropy by means of decoherence, while for $P_{\text {sub }}$ it would include an information transfer from the system to the environment (that is, an entropy transfer from the environment to the system - usually attributed to heat or noise). The "doorway channel" in this picture describes irrelevant degrees of freedom which can directly interact with the relevant channel. In the case of entanglement, it corresponds to virtual decoherence. Note, however, that, for these quantum Zwanzig projectors, $\rho_{\text {rel }}$ includes an apparent (improper) ensemble of different Everett "worlds", 
since their superposition has been dislocalized (become irrelevant to local observers). This means that the formal entropy $S\left[\rho_{r e l}\right]$ contains not only physical entropy, but also the "entropy of lacking information" about the outcomes of all measurements or spontaneous symmetry breakings. Physical entropy is defined as a function of these macroscopic quantities. Definite macroscopic (classical) histories in quantum mechanical description are thus based on a collapse or branching of the wave function, while classically they merely require the selection of subensembles, which represent incomplete information ("ignorance") about the real physical states. Such a selection is required in order to describe individual macroscopic histories whenever microscopic causes have macroscopic effects. In quantum theory, this "selection" corresponds to a genuine quantum measurement.

Explicit models for the irreversible process of decoherence and their consequences are discussed in the accompanying paper by Erich Joos.

\section{Concluding remarks}

To conclude, let me emphasize that the concept of decoherence does not contain any new physical laws or assumptions beyond the established framework of quantum theory. Rather, it is is a consequence of the universal application of quantum concepts (superpositions) and their unitary dynamics.

However, a consistent interpretation of this theory in accordance with the observed world requires a novel and nontrivial identification of observers with appropriate quantum states of local systems which exist only in certain, dynamically autonomous components of the global wave function. Accordingly, it is the observer who "splits" indeterministically - not the (quantum) world.

This interpretation is an attempt to replace the "pragmatic irrationalism" that is common in quantum theory textbooks (complementarity, dualism, uncertainty etc.) by a consistent application of those concepts which are actually, and without restriction or exception successfully, used when the theory is applied.

Acknowledgements: I wish to thank Erich Joos and Claus Kiefer for their collaboration over many years, for their persisting interest in the subject, and for their critical comments on the manuscript of this contribution.

\section{References}

[1] M. Brune, E. Hagley, J. Dreyer, X. Maitre, Y. Moali, C. Wunderlich, J.M. Raimond, and S. Haroche, Phys. Rev. Lett. 77, 4887 (1996).

[2] M. Arndt, O. Nairz, J. Vos-Andreae, C. Keler, G. van der Zouw, and A. Zeilinger, Nature 401, 680 (1999).

[3] M. Schlosshauer, Rev. Mod. Phys. 761267 (2004); Ann. Phys. (N.Y.), to be published quant-ph/0506199

[4] G. Bacciagaluppi, http://plato.stanford.edu/entries/qm-decoherence/

[5] H.D. Zeh in: Science and Ultimate Reality, J.D. Barrow, P.C.W. Davies, and C.L. Harper, edts. (Cambridge UP 2004) - quant-ph/0204088.

[6] E. Joos and H.D. Zeh, Z. Phys. B59, 223 (1985).

[7] E. Joos, H.D. Zeh, C. Kiefer, D. Giulini, J. Kupsch, and I.-O. Stamatescu, Decoherence and the Appearance of a Classical World in Quantum Theory, 2nd edn. (Springer, Berlin 2003).

[8] H.D. Zeh, The Physical Basis of the Direction of Time, 4th edn. (Springer, Berlin 2001); see also www.time-direction.de. 
[9] B. d'Espagnat, in: Preludes in theoretical physics, A. De-Shalit, H. Feshbach, and L. van Hove, edts. (North-Holland, Amsterdam 1966).

[10] H.D. Zeh, Z. Phys. 202, 38 (1967); see also Chap. 9 of [7].

[11] H.D. Zeh, Phys. Lett. A116, 9 (1986); A126, 311 (1988).

[12] W.H. Zurek, Phys. Today 44 (Oct.), 36 (1991).

[13] M. Gell-Mann and J.B. Hartle, Phys. Rev. D47, 3345 (1993).

[14] H.D. Zeh, Found. Phys. 1, 69 (1970).

[15] O. Kübler and H.D. Zeh, Ann. Phys. (N.Y.) 76, 405 (1973).

[16] E. Schrödinger, Proc. Cambridge Phil. Soc. 31, 555 (1935).

[17] R. Omnès, Phys. Rev. D 71, 065011 (2005) - quant-ph/0411201

[18] O. Ulfbeck and A. Bohr, Found. Phys. 31, 757 (2001).

[19] J.S. Bell, Speakable and Unspeakable in Quantum Mechanics (Cambridge UP 1987).

[20] J.M. Jauch, Foundations of Quantum Mechanics (Addison Wesley, Reading Mass. 1968).

[21] E. Joos, Phys. Rev. D29, 1626 (1984); see also Chap. 3 of [7].

[22] W.H. Zurek, Phys. Rev. D24, 1516 (1981); dto. D26, 1862 (1982).

[23] G. Lüders, Ann. Phys. (Leipzig) 8, 322 (1951).

[24] N. Graham, The Everett Interpretation of Quantum Mechanics (Univ. North Carolina, Chapel Hill 1970).

[25] B. d'Espagnat, edt., Foundations of Quantum Mechanics (49th Enrico Fermi School, Varenna), (Academic Press, New York 1971).

[26] J.S. Bell, Physics 1, 195 (1964).

[27] A. Peres, Phys. Rev. Lett. 77, 1413 (1996); V. Vedral, M.B. Plenio, M.A. Rippin, and P.L. Knight, Phys. Rev. Lett. 78, 2275 (1997).

[28] J.-G. Demers, and C. Kiefer, Phys. Rev. D53, 7050 (1996).

[29] D.N. Page, Phys. Rev. Lett. 44, 301 (1980).

[30] H.D. Zeh, Phys. Lett. A 347, 1 (2005) - gr-qc/0507051; C. Kiefer, gr-qc/0508120

[31] E. Joos, Phys. Lett. A116, 6 (1986).

[32] H.D. Zeh, in: Physical Origins of the Asymmetry of Time, J.J. Halliwell, J. Perez-Mercader, and W.H. Zurek, edts. (Cambridge UP 1994).

[33] C. Kiefer and H.D. Zeh, Phys. Rev. D51, 4145 (1995); C. Kiefer, Quantum Gravity, (Clarendon Press, Oxford 2004).

[34] E. Schmidt, Math. Annalen 63, 433 (1907).

[35] Ph. Pearle, Int. J. Theor. Phys. 18, 489 (1979).

[36] H.D. Zeh, Found. Phys. 3, 109 (1973) - quant-ph/0306151

[37] W.H. Zurek, S. Habib, and J.P. Paz, Phys. Rev. Lett. 70, 1187 (1993).

[38] A.O. Caldeira and A.J. Leggett, Physica 121A, 587 (1983).

[39] W.H. Zurek and J.P. Paz, Phys. Rev. Lett. 72, 2508 (1994); Nuovo Cim., 110B, 611 (1995).

[40] R. Zwanzig, Boulder Lectures in Theoretical Physics 3, 106 (1960). 